archives

of thermodynamics

Vol. 34(2013), No. 1, 71-83

DOI: 10.2478 /aoter-2013-0005

\title{
Analysis of the occurrence of natural convection in a bed of bars in vertical temperature gradient conditions
}

\author{
RAFAŁ WYCZÓŁKOWSKI \\ DOROTA MUSIAE*
}

Czestochowa University of Technology, Faculty of Process and Materials Engineering and Applied Physics, Department of Industrial Furnaces and Environmental Protection al. Armii Krajowej 19, 42-200 Częstochowa, Poland

\begin{abstract}
The reason for undertaking this study was to determine the possible involvement of natural convection in the global heat transfer, that occurs in the heated steel rods bed. This problem is related to the setting of the effective thermal conductivity of the bars bed. This value is one of the boundary conditions for heating modeling of steel rods bundles during heat treatment. The aim of this study was to determine for which geometry of the bed bars, there will be no free convection. To analyze the problem the Rayleigh criterion was used. It was assumed that for the value of the number $\mathrm{Ra}<1700$ convection in the bed bars does not occur. For analysis, the results of measurements of the temperature distribution in the unidirectionally heated beds of bars were used. It has been shown, that for obtained, during the test, differences of temperature between the surfaces of adjacent rods, convection can occur only when the diameter of the rod exceeds $18 \mathrm{~mm}$.
\end{abstract}

Keywords: Natural convection; Bed of bars; Porous material

\section{Nomenclature}

$d \quad-$ diameter, $\mathrm{m}$

$F \quad-\quad$ cross-sectional area of the gap, $\mathrm{m}^{2}$

$g \quad-$ gravitational acceleration, $\mathrm{m} / \mathrm{s}^{2}$

\footnotetext{
${ }^{*}$ Corresponding Author. E-mail: musialdt@wp.pl
} 


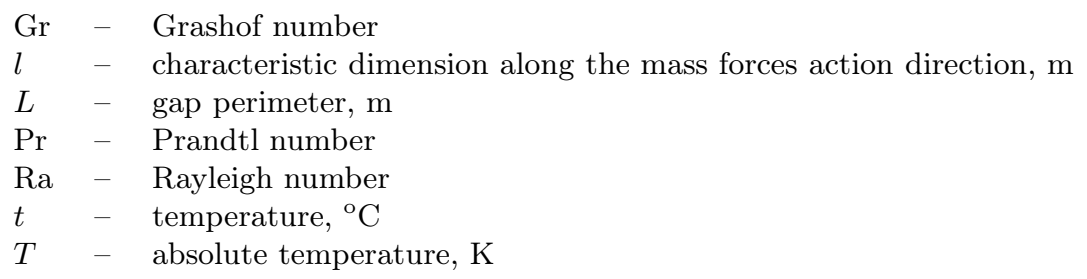

\section{Greek symbols}

$\beta-\quad$ volume coefficient of expansion, $1 / \mathrm{K}$

$\nu \quad-$ kinematic viscosity, $\mathrm{m}^{2} / \mathrm{s}$.

$$
\begin{aligned}
& \text { Subscripts } \\
& \begin{array}{lll}
a & - & \text { average } \\
B & - & \text { bar } \\
C & - & \text { cell } \\
h & - & \text { hydraulic } \\
r d & - & \text { radiative } \\
S & - & \text { square } \\
t r & - & \text { true } \\
T & - & \text { triangle }
\end{array}
\end{aligned}
$$

\section{Introduction}

Steel bars belong to basic metallurgical products obtained from the rolling process. They find wide application both as finished products and as a semifinished products to be further processed. For special applications, bars of strictly specified mechanical properties are used, which are obtained via heat or thermochemical treatment [1]. In industrial mass processes, bars to be heat treated are loaded into the furnace in the form of packed bundles of a cross-section shape similar to circular [2]. The view of such a bundle is shown in Fig. 1. This is, on the one hand, dictated by transport considerations and, on the other hand, will allow the furnace heating chamber space to be effectively used. As can be observed (Fig. 1), in the transverse direction the bar bundle constitutes an inhomogeneous system which can be treated as porous material of a grainy structure. As a consequence, a complex heat flow occurs in the cross-section of such a medium during heating. In a general case, the following heat transfer mechanisms are involved here: conduction in the bar cross-section, conduction in the medium filling the gaps (pores) formed between the bars, contact conduction at the bar contact location, radiation between bar surfaces, and convection in the gaseous medium. The above-described regime of heat flow within the bundle region causes the thermal properties of the whole bundle to deviate 
substantially from those of the bars themselves. This causes complications in the computation of the process of heating of this type of charge.

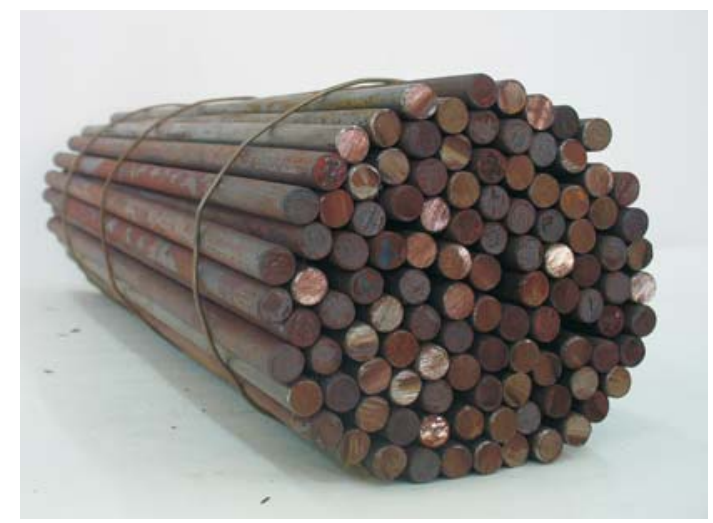

Figure 1. View of a packed bar bundle showing its porous structure.

The situation described above causes the thermal properties of a bar bundle to be expressed using the effective thermal conductivity, thanks to which the complex heat flow occurring in the porous material is treated as pure conduction, similarly as in homogeneous material [3]. The determination of the optimal time of heating a bundle of bars during heat treatment requires the knowledge of the behaviour of the effective thermal conductivity coefficient coefficient within the entire temperature range in which the process is conducted. This information can be obtained from the theoretical analyses of the heat exchange mechanisms occurring in the region of the medium under consideration. Particular difficulties are encountered in the mathematical description of convective heat transfer, because in these case the motion of fluid must be considered.

When examining a packed bar bundle it is assumed that its porosity is of a closed type. In such a situation, the heat flow associated with fluid motion has the character of natural convection within a limited space. A starting point for the theoretical analysis of this problem are the laws of conservation of mass, momentum and energy, which are expressed by appropriate differential equations for continuity, fluid motion and heat transfer $[4,5]$. The analytical solution of these equations is only possible by approximate methods, whereby results are obtained in the form of discrete distributions of the values of particular parameters at selected points of the region under consideration [6]. In the case of natural convection in an enclosed space, wall boundary layers form at the region edges, which strongly influence the 
motion of fluid within the region. The interrelation between the flow in the interior and in the wall boundary layers, as observed in this case, makes the theoretical analysis of the problem quite difficult [4-6].

The relevant literature provides several examples of theoretical and experimental analyses of the problems of natural convection in an enclosed space. The reported studies concern generally the space of a simple geometry and simple boundary conditions. Theoretical analyses of natural convection between parallel vertical surfaces and in rectangular spaces have shown that the main parameters decisive to the motion of fluid are: surface temperature difference $(T)$, distance between the surfaces, the height-towidth proportion of the region, and the value of Rayleigh number (Ra) which, for natural convection, is a criterial number.

Depending on the value of the Ra number, three flow regimes can be distinguished. The first of these regimes occurs when the Ra number value is less than 1700; the heat transfer within the fluid region takes place in this case solely by conduction. This situation is confirmed by rectilinear arrangements of isotherms in the fluid, being characteristic of this state. The second flow regime occurs with Ra number values fall roughly within the range from 1700 to 3000 . A characteristic feature of this state, which is referred to in literature as transitory, is the appearance of wall boundary layers. The flow field in this case is characterized by constant temperature values in the horizontal direction and a nearly linear temperature increase in the vertical direction. The considerable share of convection in the heat transfer is indicated by the curvilinear shape of isotherms in the majority of the fluid region. The third flow regime, called the wall boundary state, occurs with Ra number values greater than 3000. In this flow regime, the occurrence of developed wall boundary layers is observed. The thicknesses of these layers are, however, small compared to the overall fluid layer thickness. In the space limited by the parallel vertical surfaces, the thickness of the wall boundary layer on the warmer wall increases in the upward direction, while on the cooler wall - in the downward direction. After exceeding the critical value of $\mathrm{Ra}=47000$, the fluid flow in the gap changes from laminar to turbulent. The formation of turbulent pulsations is observed here, whose intensity increases with increasing Ra number. These pulsations occur primarily in the interior of the region.

The purpose of the presented analysis was to determine the maximum bar diameter for which, in a packed flat bed composed of these bars, no natural convection will occur. 


\section{Research methodology}

Subjected to analysis were two geometrical bar arrangements: covered and partitioned, whose schematic diagrams are shown in Fig. 2. It was assumed that the beds had a temperature gradient directed vertically upwards, and the fluid filling the gaps between the bars was air. For such assumptions, convection in bed gaps would occur when the following condition was satisfied namely $\mathrm{Ra} \geq 1700$.

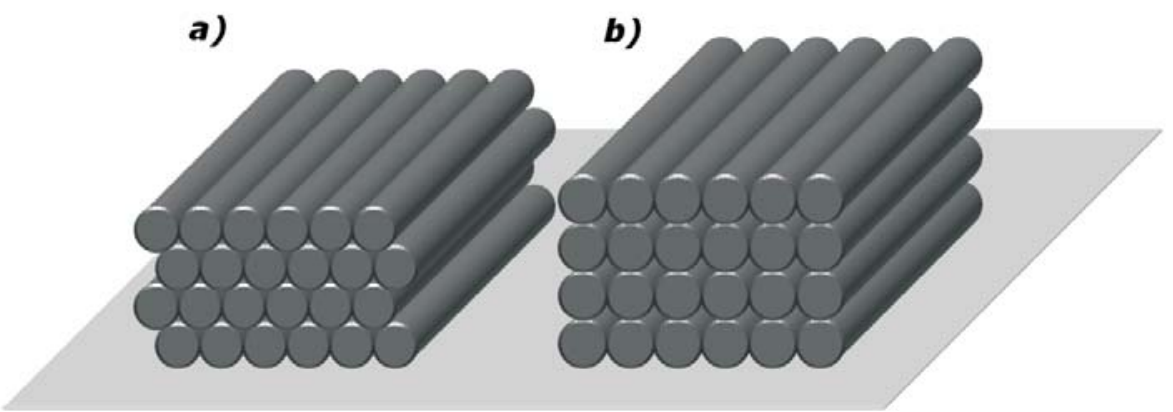

Figure 2. Bar beds taken for the analysis: a) a bed with a partitioned layer arrangement b) a bed with a covered layer arrangement.

Writing the Rayleigh number of the product of two other criterial numbers, namely the Grashof number (Gr) and the Prandtl number (Pr)

$$
\mathrm{Ra}=\mathrm{GrPr}=\frac{\beta g l^{3}}{\nu^{2}} \Delta t \operatorname{Pr}=\frac{\beta g l^{3}}{\nu^{2}} \Delta t \frac{\nu}{a},
$$

where $\Delta t$ is the temperature difference of bars surfaces in an adjacent layers of the bed. We obtain the following condition

$$
\frac{\beta g l^{3}}{\nu^{2}} \Delta t \frac{\nu}{a} \geq 1700
$$

For air which, according to the assumption, fills the bed gaps, the value of the Prandtl number does not depend on the temperature and equals 0.7 [7]. In addition, it is assumed that the volume coefficient of expansion is equal to

$$
\beta=1 / T_{a},
$$

where $T_{a}$ denotes the average gas temperature in the absolute temperature scale. The condition (2) can be therefore written in the following form:

$$
0.7 \frac{g l^{3}}{T_{a} \nu^{2}} \Delta t \geq 1700 \text {. }
$$


If the space of the gap under consideration had a cylindrical shape, then its characteristic dimension would be the diameter $d$ of the bar. In that case, considering the gap diameter, the convective motion of the fluid will occur after the following condition has been satisfied:

$$
d \geq \sqrt[3]{\frac{1700 \nu^{2} T_{a}}{0.7 g \Delta t}}
$$

The value of the diameter, as calculated from relationship (5) can be called the limiting diameter, as only after exceeding this particular value will the convective air movements come to occur in the region under analysis. Due to the fact that the gaps in bar beds do not have a cylindrical shape, the hydraulic mean diameter $d_{h}$ should be used, defined by the equation

$$
d_{h}=\frac{4 F}{L}
$$

The aim of the conducted analysis requires, therefore, that the relationship between the bar diameter and the hydraulic diameter of the gap be determined for the beds under consideration. The shapes of beds for the partitioned and the covered beds of bars are demonstrated in Fig. 3. The presented arrangements constitute elementary heat exchange cells for par-
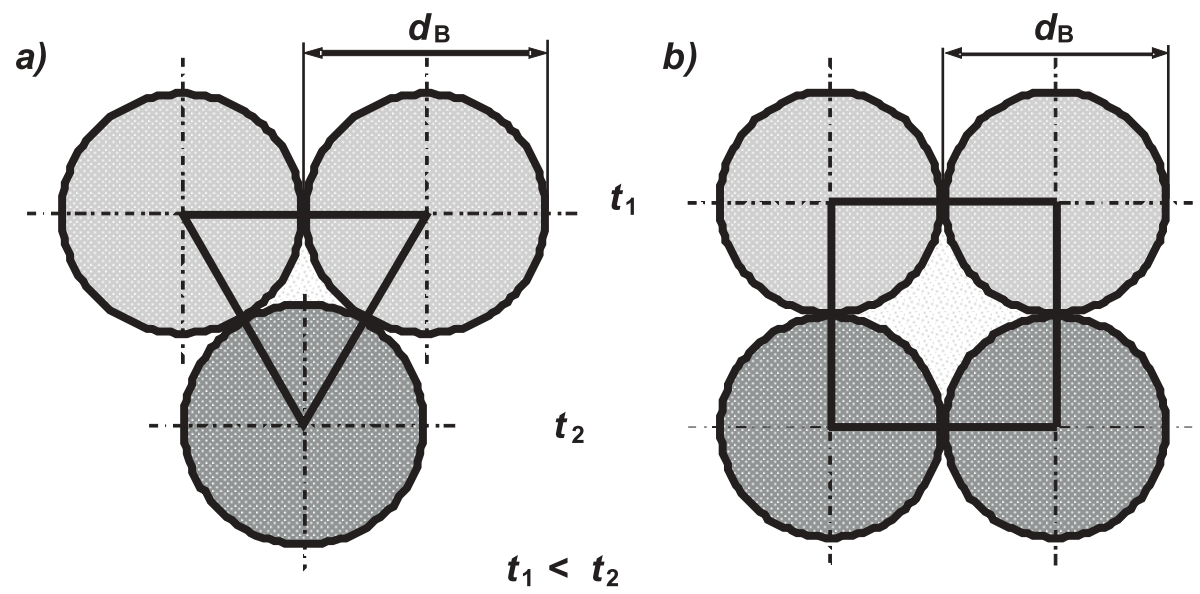

Figure 3. Schematic diagrams of elementary cells: a) for the partitioned bed - C1 cell; b) for the covered bed - C2 cell.

ticular geometric cases. In the further discussion, the cell for the partitioned 
arrangement will be denoted with the symbol $\mathrm{C} 1$, and the cell for the covered arrangement, with $\mathrm{C} 2$. According to the assumption made previously on the temperature field in the beds, the temperature of the bottom bars $\left(t_{2}\right)$ is higher than the temperature of the top bars $\left(t_{1}\right)$. It is in this case that the convective motion of the fluid will start occurring in the gap region.

For the $\mathrm{C} 1$ cell, the gap area $\left(F_{C 1}\right)$ is calculated by the subtracting the area $\left(F_{B-C 1}\right)$ of the part cross-sections bars contained in the equilateral triangle with the side equal to the bar diameter from the area of this triangle $\left(F_{T}\right)$.

$$
F_{C 1}=F_{T}-F_{B-C 1}
$$

where

$$
\begin{gathered}
F_{T}=0.433 d_{B}^{2}, \\
F_{B-C 1}=0.5 \frac{\pi d_{B}^{2}}{4}=\frac{\pi d_{B}^{2}}{8}=0.392 d_{B}^{2}
\end{gathered}
$$

so

$$
F_{C 1}=0.04 d_{B}^{2}
$$

where $d_{B}$ is the bar diameter. The gap perimeter for the $\mathrm{C} 1$ cell is:

$$
L_{C 1}=0.5 \pi d_{B}=1.57 d_{B} .
$$

Hence, the following relationship exists between the bar diameter $d_{B}$ and the gap hydraulic diameter $d_{h C 1}$ for the $\mathrm{C} 1$ cell:

$$
d_{h-C 1}=\frac{4 \cdot 0.04 d_{B}^{2}}{1.57 d_{B}}=0.1 d_{B}
$$

To calculate the gap area for the $\mathrm{C} 2$ cell, the surface area of bars occurring within the square of a side length $d_{B}$ is to be subtracted from the area of this square

$$
F_{C 2}=F_{S}-F_{B-C 2},
$$

where

$$
F_{S}=d_{B}^{2},
$$

and

$$
F_{B-C 2}=\frac{\pi d_{B}^{2}}{4}=0.785 d_{B}^{2},
$$

SO

$$
F_{C 2}=0.215 d_{B}^{2}
$$


The gap perimeter in the $\mathrm{C} 2$ cell is

$$
L_{C 2}=\pi d_{B} .
$$

For the $\mathrm{C} 2$ cell, the relationship between the bar diameter $d_{B}$ and the gap hydraulic diameter $d_{h-C 2}$ is as follows:

$$
d_{h-C 2}=\frac{4 \cdot 0.215 d_{B}^{2}}{3.14 d_{B}}=0.27 d_{B} .
$$

By substituting Eqs. (12) and (18) in relationship (5), direct formulas for the limiting bar diameter are obtained. These take on the following forms:

$$
d_{B-C 1}=10 \sqrt[3]{\frac{1700 \nu^{2} T_{a}}{0.7 g \Delta t}} \text { for the partitioned bed bundle },
$$

and

$$
d_{B-C 2}=3.7 \sqrt[3]{\frac{1700 \nu^{2} T_{a}}{0.7 g \Delta t}} \text { and for the covered bed bundle }
$$

where

$$
\Delta t=t_{2}-t_{1} .
$$

As follows from relationships (19) and (20), the limiting diameter for the $\mathrm{C} 1$ cell is greater 2.7 than that for the $\mathrm{C} 2$ cell.

\section{Computation results}

To carry out computation, the value of the temperature difference $(\Delta t)$ between the bar surfaces limiting the considered gaps must be assumed. It is essential at this point to know how this parameter behaves with increasing temperature under real bar heating conditions. Not having this information available, we performed preliminary computations for four constant values of the parameter $\Delta t\left(10,50,100\right.$ and $\left.200{ }^{\circ} \mathrm{C}\right)$.

Another quantity that influences the computation outcome is the kinematic viscosity of the medium filling the gap. For air, information on the variation of kinematic viscosity as a function of temperature was taken from literature [8]. Based on these values, the limiting diameters were computed for a temperature from 0 to $1000{ }^{\circ} \mathrm{C}$. The computation results are shown in Fig. 4. 
a)

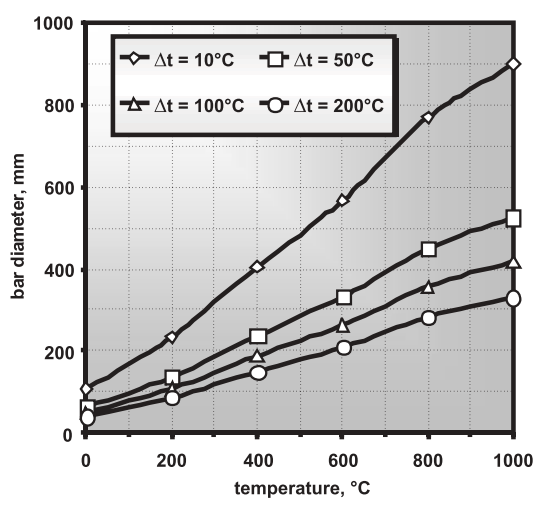

b)

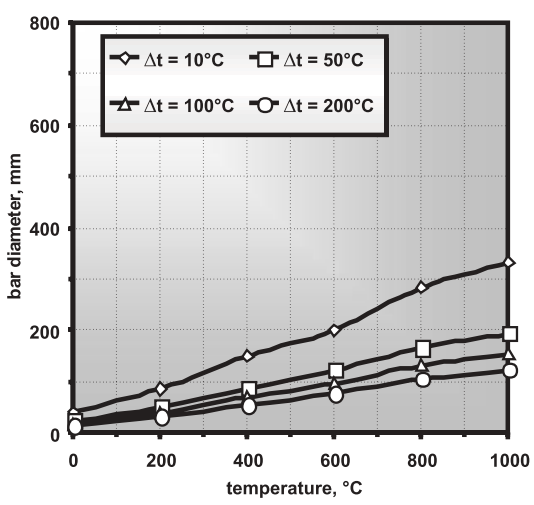

Figure 4. Results of the preliminary computation of the limiting bar diameter: a) for the $\mathrm{C} 1$ cell; b) for the $\mathrm{C} 2$ cell.

As can be observed, the value of the limiting bar diameter for both cells increases nearly linearly as a function of air temperature. Moreover, the value of the $d_{B-C}$ parameter is strongly influenced by the temperature difference between the gap limiting surfaces. Therefore, from the point of view of reliability and precision of computation, the knowledge of the behaviour of the actual temperature field in the region of the bar bed as it is heated will be necessary. This knowledge can only be acquired by carrying out appropriate experimental tests. To this end, an experimental research was carried out, which consisted in recording the variations of temperature at selected points of appropriately prepared beds of bars as they were heated in an electric laboratory oven. Two bar beds were tested, each of them being $120 \mathrm{~mm}$ wide and $350 \mathrm{~mm}$ long, and built of four layers of $20 \mathrm{~mm}$ diameter bars, as shown in Fig. 5. One bed was made up of a bar laid in a covered manner, while the other was a bar arranged in a partitioned manner. The construction of the oven enabled a single-dimension temperature field to be obtained within the charges being heated, with the temperature gradient being oriented vertically.

Temperature measurements were taken on the surfaces of bars lying between the second and the third layers at points indicated in Fig. 6. C-type $0.5 \mathrm{~mm}$-diameter jacket thermocouples were used for the measurements.

The experiment was carried out for three samples, and the averaged measurement result is shown in Fig. 7. Figure 7a represents variations in the average temperature of gaps as a function of heating time. In the first 

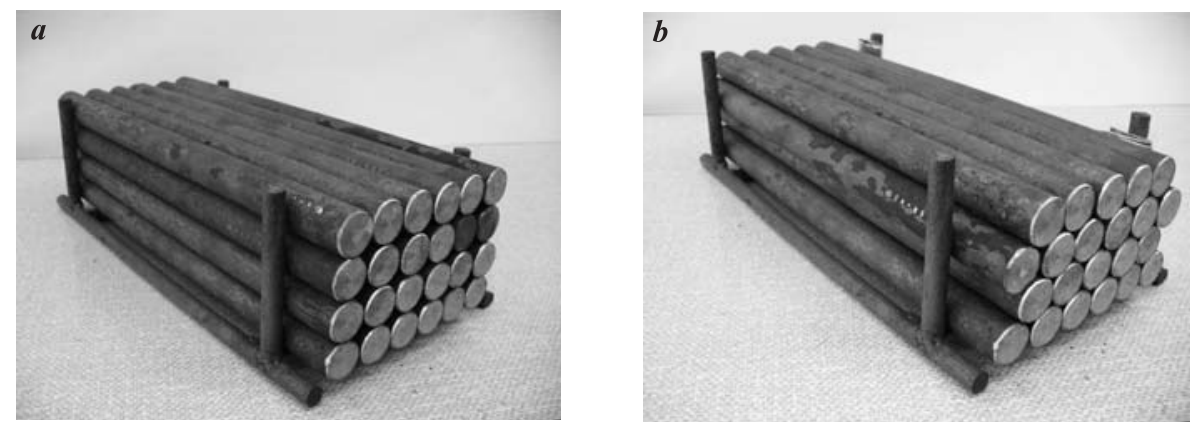

Figure 5. View showing bar beds subjected to heating: a) a covered bundle; b) a partitioned bundle.

a)

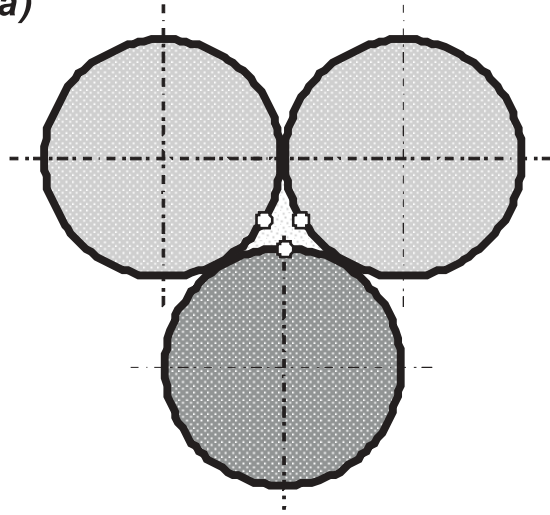

b)

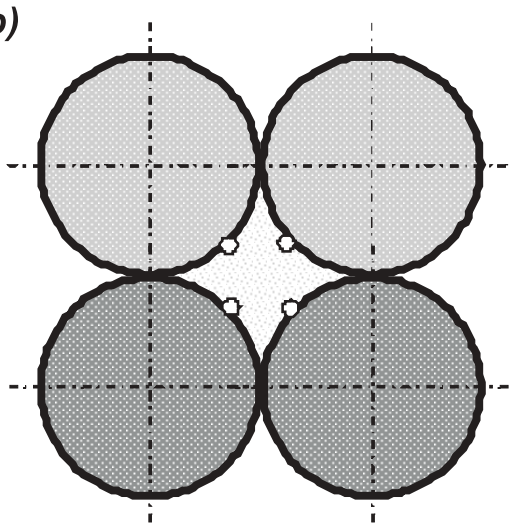

Figure 6. Temperature measurement points at bar surfaces: a) for the partitioned bed; b) for the covered bed.

phase of the process, the value of this parameter increases almost linearly for both charges. In the subsequent part, because of the equalization of the temperature field within the charge, the average temperatures started approaching asymptotically the ultimate oven temperature, which in the tests under consideration was $850{ }^{\circ} \mathrm{C}$. Figure $7 \mathrm{~b}$ represents variations in the temperature differences within charge gaps (cells) as a function of the average cell temperature. As can be seen from the shape of the lines in this diagram, the results for both cases exhibit a nearly linear behaviour. Using the least squares method, regression functions were determined for the charged tested to describe the relationship between the temperature difference within the gaps and the average temperature. On this basis, the 
a)

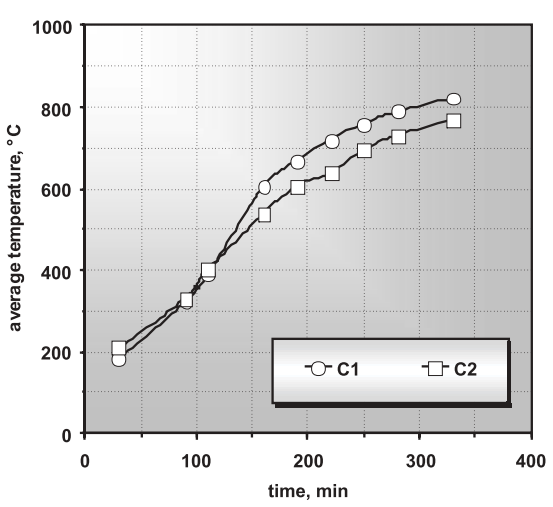

b)

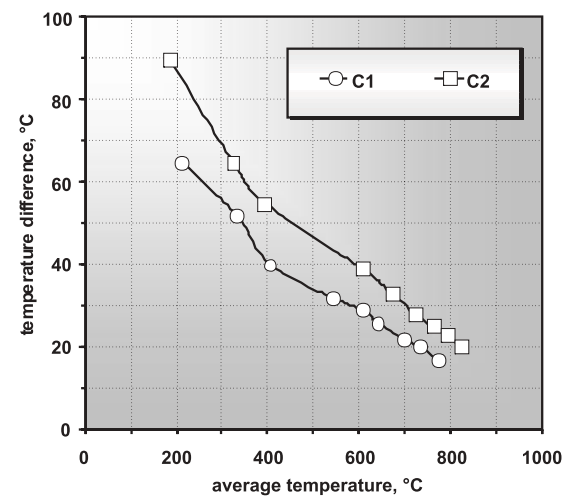

Figure 7. Results of experimental tests: a) variation of the average temperatures of the cells $\mathrm{C} 1$ and $\mathrm{C} 2$ as a function of time; b) variations of the temperature differences as a function of the average temperature of the cells under consideration.

following relationships were obtained:

$$
\begin{aligned}
& \text { for the covered bundle: } \Delta t_{1}=-0.110 t-109.83 \text {, } \\
& \text { for the partitioned bundle: } \Delta t_{2}=-0.107 t-96.23 \text {. }
\end{aligned}
$$

These are decreasing functions, as the temperature differences decrease as the charge temperature increases. This results from the fact that the temperature field within the charge equalizes as the heating progresses.

Using the empirical relationships (22) and (23), the limiting bar diameter values for the cells $\mathrm{C} 1$ and $\mathrm{C} 2$ were computed for the temperature range from 0 to $900{ }^{\circ} \mathrm{C}$. The results of these computations are shown in Fig. 8. The line in this diagram represents an exponential function. The regression functions for individual cells assume here the following forms:

$$
\begin{aligned}
& \text { for the covered bundle: } \quad d_{C 1}=61.772 e^{0.0031 t}, \\
& \text { for the partitioned bundle: } d_{C 2}=21.366 e^{0.0031 t} .
\end{aligned}
$$

From the obtained results it can be inferred that in the case of heating a bundle of bars in a partitioned arrangement the natural convection will never occur, if the bar diameter is less than $54 \mathrm{~mm}$. For the covered bundle this value is $18 \mathrm{~mm}$. For example, for a temperature of $200^{\circ} \mathrm{C}$, the limiting diameter values will be, respectively:

the partitioned bundle $d_{C 1}=127 \mathrm{~mm}$,

the covered bundle $\quad d_{C 2}=43 \mathrm{~mm}$. 


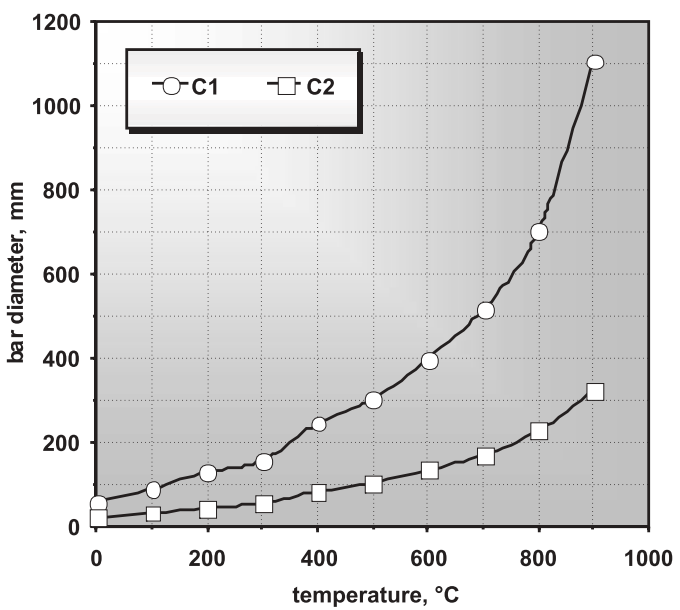

Figure 8. Temperature dependence of the limiting bar diameters values for the cells $\mathrm{C} 1$ and $\mathrm{C} 2$.

So, if a bundle composed of $130 \mathrm{~mm}$-diameter bars is subjected to heating, convection within the gaps will only occur in the initial period of the process up to the point when the charge temperature reaches a level of approx. $200{ }^{\circ} \mathrm{C}$. The annealing heat treatment of steel bars is conducted at a temperature of about $800^{\circ} \mathrm{C}$. It can be seen from the obtained results that, in the entire temperature range of this process, the convection will occur in the bundle gaps, if the bar diameter is slightly greater than $200 \mathrm{~mm}$ (for the covered bundle). Most often, however, bars in bundles are subjected to heat treatment for a diameter range from 5 to $25 \mathrm{~mm}[2,9]$. In such a situation, when analyzing the heat transfer within the bundle, the convection of the gaseous medium in the gaps formed between the bars can be neglected.

\section{Summary}

On the basis of the performed tests and computations it has been demonstrated that during heating of bars in the form of bundles, for specific conditions of bar diameters and temperature differences, the convective motion of gas in the gaps will not occur. The shape of gaps, which is dependent on the bar arrangement, is also important here.

Thanks to this, in the analysis of heat transfer in a bed of bars, should be taken into account only the phenomena associated with heat conduction and 
radiation. It should be noted, that the heat transfer in this case concerns both phases of the bed and contact conduction which take place in the contact spots of the bars.

Received 1 March 2011

\section{References}

[1] Dobrzański L.A.: Metallic Engineering Materials. WNT, Warsaw 2004 (in Polish).

[2] SAHAY S.S., KRISHnAN K.: Model based optimization of continuous annealing operation for bundle of packed rods. Ironmak. Steelmak., 34( 2007), 1, 89-94.

[3] Kaviany M.: Principles of Heat Transfer in Porous Media. Springer-Verlag, New York 1991.

[4] Pastucha L., Otwinowski W.: Basis of Heat Transfer. Wydawnictwo Politechniki Częstochowskiej, Częstochowa 1999 (in Polish).

[5] Wiśniewski S.: Heat Transfer. PWN, Warsaw 1979 (in Polish).

[6] WyczóŁkowski S.: Modelling of Steady Free Convection in Two-dimensional Enclosed Space. Wydawnictwo Politechniki Częstochowskiej, Częstochowa 1998 (in Polish).

[7] Kostowski E.: Heat Flow. Wydawnictwo Politechniki Śląskiej, Gliwice 2000 (in Polish).

[8] Raźnjević K.: Thermal Table with Diagrams. WNT, Warsaw 1966 (in Polish).

[9] Musią D. Heat Transfer in Steel Rods Bundle During Heating. PhD thesis, Czestochowa University of Technology. Częstochowa 2007 (in Polish). 